\title{
Patient-derived Mammosphere and Xenograft Tumour Initiation Correlates with Progression to Metastasis
}

\author{
Rachel Eyre $^{1}$ • Denis G. Alférez ${ }^{1}$ - Kath Spence ${ }^{1}$ Mohamed Kamal ${ }^{1,2}$. \\ Frances L. Shaw ${ }^{1}$ • Bruno M. Simões ${ }^{1}$ • Angélica Santiago-Gómez ${ }^{1}$. \\ Aida Sarmiento-Castro ${ }^{1} \cdot$ Maria Bramley $^{3} \cdot$ Mohammed Absar $^{3} \cdot$ Zahida Saad $^{4}$. \\ Sumohan Chatterjee $^{4}$ - Cliona Kirwan ${ }^{5}$ - Ashu Gandhi ${ }^{5}$ - Anne C. Armstrong ${ }^{6}$. \\ Andrew M. Wardley ${ }^{6}$ - Ciara S. O'Brien ${ }^{6}$ • Gillian Farnie ${ }^{7}$ - Sacha J. Howell ${ }^{8,6}$. \\ Robert B. Clarke ${ }^{1}$
}

Received: 25 May 2016 / Accepted: 5 September 2016 /Published online: 28 September 2016

(C) The Author(s) 2016. This article is published with open access at Springerlink.com

\begin{abstract}
Breast cancer specific mortality results from tumour cell dissemination and metastatic colonisation. Identification of the cells and processes responsible for metastasis will enable better prevention and control of metastatic disease, thus reducing relapse and mortality. To better understand these processes, we prospectively collected 307 patient-derived breast cancer samples $(n=195$ early breast cancers (EBC) and $n=112$ metastatic samples $(\mathrm{MBC})$ ). We assessed colony-forming activity in vitro by

The original version of this article was revised: Table 1 contained the following errors: "Grade" was incorrectly labelled as "Grade 1", "Nodal Involvement" was incorrectly labelled as "Hormone Receptor Status", the ER positive and ER negative labels were reversed, the Her2 positive was labelled as "Po" instead of "Pos", and "Endocrine therapy" was labelled as" "Endoctrine therapy". In Table 2, "Grade" was incorrectly labelled as "Grade 1", and "Hormone Receptor Status" was incorrectly labelled as "Nodal Involvement". In Table 3, "Pathology" was incorrectly labelled as "Pathlofy".
\end{abstract}

Rachel Eyre and Denis G. Alférez contributed equally to this work.

Electronic supplementary material The online version of this article (doi:10.1007/s10911-016-9361-8) contains supplementary material, which is available to authorized users.

Robert B. Clarke

robert.clarke@manchester.ac.uk

1 Breast Biology Group, Breast Cancer Now Research Unit, Division of Molecular and Clinical Cancer Sciences, Manchester Cancer Research Centre, University of Manchester, Wilmslow Road, Manchester M20 4QL, UK

2 Department of Zoology, Faculty of Science, University of Benha, Benha, Egypt

3 Pennine Acute Hospitals NHS Trust, Manchester, UK

4 Salford Royal NHS Foundation Trust, Manchester, UK growing isolated cells in both primary (formation) and secondary (self-renewal) mammosphere culture, and tumour initiating activity in vivo through subcutaneous transplantation of fragments or cells into mice. Metastatic samples formed primary mammosphere colonies significantly more frequently than early breast cancers and had significantly higher primary mammosphere colony formation efficiency ( $0.9 \%$ vs. $0.6 \%$; $p<0.0001)$. Tumour initiation in vivo was significantly higher in metastatic than early breast cancer samples (63\% vs. $38 \%$,

5 University Hospitals of South Manchester NHS Foundation Trust, Manchester, UK

6 The Christie NHS Foundation Trust, Wilmslow Road, Manchester M20 4BX, UK

Cancer Stem Cell Research, Division of Molecular and Clinical Cancer Sciences, Manchester Cancer Research Centre, University of Manchester, Wilmslow Road, Manchester M20 4QL, UK

8 Breast Cancer Now Research Unit, Division of Molecular and Clinical Cancer Sciences, Manchester Cancer Research Centre, University of Manchester, Wilmslow Road, Manchester M20 4QL, UK 
$p=0.04)$. Of 144 breast cancer samples implanted in vivo, we established 20 stable patient-derived xenograft (PDX) models at passage 2 or greater. Lung metastases were detected in mice from 14 PDX models. Mammosphere colony formation in vitro significantly correlated with the ability of a tumour to metastasise to the lungs in vivo $(p=0.05)$, but not with subcutaneous tumour initiation. In summary, the breast cancer stem cell activities of colony formation and tumour initiation are increased in metastatic compared to early samples, and predict metastasis in vivo. These results suggest that breast stem cell activity will predict for poor outcome tumours, and therapy targeting this activity will improve outcomes for patients with metastatic disease.

Keywords Breast cancer · Patient-derived xenografts . Metastasis $\cdot$ Mammosphere $\cdot$ Stem cell activity

\section{Introduction}

Despite advances in breast cancer diagnoses and treatment, $20 \%$ of patients will develop metastatic tumours at distant sites, eventually leading to death [1]. Metastasis is a multistep process involving local invasion, intravasation, survival in the circulation, extravasation and colonisation at secondary sites [2]. Although much research has concentrated on understanding the metastatic process, little is known about the cells within a tumour which are able to successfully colonise distant organs. Understanding which cells drive the colonisation and growth of breast cancer cells at distant sites should lead to improvements in adjuvant therapies, designed to prevent the development of secondary tumours.

Much research has focussed on a subset of tumour cells termed cancer stem cells (CSCs), which are capable of selfrenewal, and are responsible for tumour initiation [3, 4]. Several methods are used to isolate CSCs and assess their activity, including both functional assays and the expression of cellular markers. Tumour formation following transplantation in vivo is considered the gold standard assay to measure CSC activity, and the mammosphere colony forming assay is widely used in vitro. This assay was first developed to quantify neural stem cells [5], and was later demonstrated to isolate a stem cell population in mammary tissue [6]. It has since been used to measure cancer stem cell activity in both DCIS and invasive ductal carcinomas $[7,8]$.

There is evidence that breast cancer cells with a stem celllike phenotype are metastasis-forming cells in breast cancer. Breast cancer stem-like cells isolated from cell lines are more frequently metastatic compared to non-CSC populations when injected into immunocompromised mice [9, 10]. Furthermore, tumour ALDH1 expression, a marker of breast CSCs, has been shown to be an independent predictive factor for early metastasis in patients [9]. Most recently, single cell analysis of metastatic cells in patient-derived xenograft models revealed that these possessed a stem cell-like gene signature [11]. However the role of CSCs in the metastatic behaviour of patient-derived breast cancer samples is yet to be determined.

Using 307 patient-derived samples from early $(n=195$, $(\mathrm{EBC}))$ and metastatic $(n=112,(\mathrm{MBC}))$ breast cancers, we evaluated the role of CSC activity in breast cancer metastasis. We assessed both mammosphere colony formation in vitro and tumour initiation in vivo to determine the relationship between CSC activity and disease progression. Tumour implantation in vivo resulted in the production of patient-derived xenograft (PDX) models, and metastasis in these models was correlated to CSC activities in the patient-derived samples. We show that both mammosphere formation in vitro and tumour take in vivo are increased in metastatic samples compared to early breast cancer samples, and mammosphere formation in vitro predicts for metastasis to the lung in PDX models in vivo. We thus conclude that cancer stem cell activity in vitro correlates with metastasis in vivo, and that the mammosphere assay should be further investigated as a tool for identifying metastasis preventing drugs.

\section{Materials and Methods}

\section{Sample Collection}

195 early breast cancer surgical specimens were collected by the Manchester Cancer Research Centre Biobank from patients undergoing surgery for primary tumour removal at University Hospital of South Manchester, Salford Royal and The Pennine Acute Hospitals NHS Trusts. 112 unrelated metastatic samples (pleural effusion or ascitic fluid) were collected from patients during standard therapeutic drainage procedures at The Christie NHS Foundation Trust. All patients underwent fully informed consent as either "basic consent" or "animal consent" in accordance with local research ethics committee guidelines (see Compliance with Ethical Standards section for consenting procedures). Clinical information for samples used in this study (grade, nodal involvement, oestrogen receptor (ER) status, Her2 receptor status, radiotherapy, chemotherapy and endocrine therapy prior to sample collection, Nottingham Prognostic Index (NPI) score) is detailed in Table 1. Cells from samples with basic consent were isolated (see below) and cancer stem cell activity was assessed in vitro only. Samples with animal consent were also implanted into mice (see Compliance with Ethical Standards section for animal ethics approvals) to assess tumour initiation in vivo (See Supplementary Fig. 1 for sample pathway).

\section{In vivo Implantation}

120 early breast cancer samples and 24 metastatic breast cancer samples were implanted into female NSG (NOD.Cg- 
Table 1 Summary of clinical data for patients included in the study. 307 patients

(195 early and 112 metastatic) were included. A summary of their clinical characteristics is shown. NA; data not available

\begin{tabular}{|c|c|c|c|}
\hline & & $\mathrm{EBC}(n=195)$ & $\operatorname{MBC}(n=112)$ \\
\hline \multirow[t]{4}{*}{ Grade } & 1 & $8(4 \%)$ & $5(4.5 \%)$ \\
\hline & 2 & $83(42.6 \%)$ & $46(41.1 \%)$ \\
\hline & 3 & $95(48.7 \%)$ & $34(30.4 \%)$ \\
\hline & NA & $9(4.6 \%)$ & $27(24.1 \%)$ \\
\hline \multirow[t]{3}{*}{ Nodal involvement } & Yes & $79(40.5 \%)$ & NA \\
\hline & No & $95(48.7 \%)$ & NA \\
\hline & NA & $21(20.7 \%)$ & NA \\
\hline \multirow[t]{3}{*}{ ER } & Pos & $133(68.2 \%)$ & $81(72.3 \%)$ \\
\hline & Neg & $58(29.7 \%)$ & $24(21.4 \%)$ \\
\hline & NA & $4(2.1 \%)$ & $7(6.3 \%)$ \\
\hline \multirow[t]{3}{*}{ Her2 } & Pos & $33(16.9 \%)$ & $10(8.9 \%)$ \\
\hline & Neg & $148(75.9 \%)$ & $91(81.3 \%)$ \\
\hline & NA & $14(7.2 \%)$ & $11(9.8 \%)$ \\
\hline \multirow[t]{2}{*}{ Radiotherapy } & Yes & $4(2.1 \%)$ & NA \\
\hline & No & $191(97.9 \%)$ & NA \\
\hline \multirow[t]{3}{*}{ Chemotherapy } & Yes & $8(4.1 \%)$ & $68(60.7 \%)$ \\
\hline & No & $187(95.9 \%)$ & $17(15.2 \%)$ \\
\hline & NA & $0(0 \%)$ & $27(24.1 \%)$ \\
\hline \multirow[t]{3}{*}{ Endocrine therapy } & Yes & $25(12.8 \%)$ & $72(64.3 \%)$ \\
\hline & No & $170(87.2 \%)$ & $13(11.6 \%)$ \\
\hline & NA & $0(0 \%)$ & $27(24.1 \%)$ \\
\hline \multirow[t]{3}{*}{ NPI } & Good $(<3.4)$ & $20(10.3 \%)$ & NA \\
\hline & Moderate/Poor (>3.4) & $152(77.9 \%)$ & NA \\
\hline & NA & $23(11.8 \%)$ & NA \\
\hline
\end{tabular}

Prkdcscid Il2rgtm $1 \mathrm{Wjl} / \mathrm{SzJ}$ ) mice in accordance with the UK Home Office Animals (Scientific Procedures) Act 1986. All tumours were implanted subcutaneously bilaterally into at least two mice per patient sample, either fresh or following one freeze/thaw. Early breast cancers were implanted as $2 \times 2 \mathrm{~mm}^{3}$ fragments, and metastatic samples were injected as $1 \times 10^{6}$ isolated cancer cells. Metastatic cells were injected in $100 \mu \mathrm{l}$ of a 50:50 mix of matrigel and mammosphere media (components detailed below). The addition of matrigel to early breast cancer fragments was tested but this did not improve tumour take rate, therefore early breast cancers were implanted without matrigel. The implantation procedure did not change during the duration of the study. Oestrogen supplementation was provided in drinking water for mice with ER positive tumours at a concentration of $8 \mu \mathrm{g} / \mathrm{ml}$. Tumour growth was measured twice weekly using callipers. When tumours reached $1.3 \mathrm{~cm}^{3}$ mice were culled and tissue fragments were either implanted into a further generation of mice, frozen for later use, or fixed and examined histologically.

\section{In vitro Sample Processing}

Early breast cancers were disaggregated by mincing with a scalpel, prior to digestion in $4.7 \mathrm{ml}$ RPMI medium plus enzymes from the Miltenyi tumour dissociation kit (130-095-
929) on a rotating platform at $150 \mathrm{rpm}$ for $2 \mathrm{~h}$. Optimisation was carried out by splitting tumours in half and comparing the following methods: rotating platform vs. gentleMACS tubes, $2 \mathrm{~h}$ digestion vs. overnight digestion, Miltenyi tumour digestion enzymes vs. collagenase. In all cases the number of viable cells (assessed using Trypan Blue on a haemocytometer) were counted following digestion. Two hours digestion on a rotating platform with Milteniyi tumour digestion enzymes was found to be optimal. Following digestion, cells were strained through a $70 \mu \mathrm{M}$ filter (BD, 352340), using a plunger from a $5 \mathrm{ml}$ syringe (Terumo, SS05SE1) to very gently massage the undigested tissue over the filter, and the filter was then rinsed with $3 \times 1 \mathrm{ml}$ RPMI medium. Cells were then further strained through a $40 \mu \mathrm{M}$ filter (BD, 352340), the filter was rinsed with $3 \times 1 \mathrm{ml} \mathrm{RPMI}$ medium and centrifuged at $1000 \mathrm{~g}$ for $5 \mathrm{~min}$ at $4{ }^{\circ} \mathrm{C}$ to pellet cells. Supernatant was removed and the cell pellet resuspend in $100 \mu \mathrm{l}$ of ice-cold PBS. Live cells were counted using Trypan Blue (Gibco, 15,250-061) on a haemocytometer, and cells were then cultured as mammospheres (see below). Metastatic samples were first centrifuged at $1000 \mathrm{~g}$ for $10 \mathrm{~min}$ to pellet cells. Pellets were resuspended in PBS and blood cells were removed by centrifugation of the cell suspension through 0.5 volumes of Lymphoprep solution (Axis 
Shield, Dundee, UK) at $800 \mathrm{~g}$ for $20 \mathrm{~min}$. Epithelial cells were removed from the interface and diluted with PBS before centrifugation at $800 \mathrm{~g}$ for $2 \mathrm{~min}$ at $4{ }^{\circ} \mathrm{C}$ to pellet cells. The supernatant was removed and a cell count performed using Trypan Blue on a haemocytometer. Cells were then cultured as mammospheres (see below).

\section{Mammosphere Assay}

Mammosphere culture was performed as previously described [12]. A single cell suspension was prepared by manual disaggregation (25 gauge needle) and a total of 500 cells $/ \mathrm{cm}^{2}$ were plated in appropriate polyHEMA (Poly (2hydroxyethylmethacrylate)) coated tissue culture plates in mammosphere medium (phenol red-free DMEM/F12 (Gibco, 21,041)) containing B27 supplement (no vitamin A; Invitrogen, 12,587), rEGF (20 ng/ml; Sigma, E-9644) and Pen-Strep). Methylcellulose was not added to mammosphere media. Cells were cultured for seven days before mammospheres with diameter greater than $50 \mu \mathrm{m}$ were counted by visual inspection at $\times 40$ magnification using a microscope fitted with a graticule. Percentage primary mammosphere-forming efficiency (MFE) was calculated by dividing the number of mammospheres formed by the number of cells plated and expressed as a percentage. To assess selfrenewal, mammospheres were counted, centrifuged $(115 \times \mathrm{g})$, and dissociated into a single cell suspension by incubation for 2 min at $37^{\circ} \mathrm{C}$ in trypsin EDTA $0.125 \%$ (Sigma), followed by mechanical dissociation (25 gauge needle). Single cells were re-plated at 500 cells $/ \mathrm{cm}^{2}$ and the number of secondary mammospheres counted after 7 days. Mammosphere selfrenewal was calculated by dividing the number of secondary mammospheres formed by the number of primary mammospheres formed.

\section{Immunohistochemistry}

All tumours/cell pellets were fixed in formalin and paraffin embedded. Tumour staining for ER, PR, Ki67 and Her2 was performed in The Christie Hospital Pathology Department. Antibodies used were anti-ER $\alpha$ (Thermo, SP1), anti-PgR (Dako, M3569), anti-Ki67 (Dako M7240) and anti-Her2 (Vector Laboratories, VP-C380). Antigen retrieval was performed either using Target Retrieval Solution $\mathrm{pH} 9$ (Dako S2367, for ER, PR and Ki67) or in $10 \mathrm{mM}$ Citrate buffer (Her2). Antibodies were detected using Dako EnVision Detection System Peroxidase/DAB, Rabbit/Mouse (Dako, K5007) and sections were counterstained with haematoxylin. Hormone receptor positivity was defined as follows; oestrogen receptor (ER) $>5 \%$ positive cells, progesterone receptor $(\mathrm{PR})>5 \%$ positive cells, Her2 receptor $3+$ or $2+$ confirmed by positive FISH analysis. Human cells were detected in PDX models using a human specific anti- mitochondrial antibody (Abcam, ab92824). Lungs, livers, femurs and lymph nodes were examined for metastases postmortem in each mouse where subcutaneous tumour growth (P1) was seen. $5 \mathrm{x}$ step sections $(40 \mu \mathrm{M})$ of each organ were stained with human specific mitochondrial antibody. Sections were stained on a Leica Bond system using standard protocol $\mathrm{F}$ and primary antibody conditions of 1:1000 for $15 \mathrm{~min}$. Metastases were detected by visual examination of stained sections. Organs where at $\geq 1$ human cell was observed were classed as metastases positive.

\section{Statistical Analysis}

Statistical analysis was performed using SPSS. Data are represented as mean \pm SEM. Statistical significance was measured using parametric testing, assuming equal variance, in the majority of experiments with standard t-tests for twopaired samples used to assess difference between test and control samples. Chi squared analysis was used for categorical variables, The Mann-Whitney U test was used for data which was not normally distributed. Differences were considered statistically significant if the probability value (p) was $\leq 0.05$.

\section{Results}

\section{Breast Cancer Stem Cell Activity is Increased in Metastatic Breast Cancer Samples}

In total, 307 patient-derived samples were collected during this study. These comprised 195 early breast cancers and 112 unrelated metastatic breast cancers. For samples with animal consent, fragments were first removed for implantation before the remainder of the sample was digested for in vitro experiments. For samples with basic consent, the whole sample was digested for in vitro experiments. Following this sample pathway, 144 samples in total were implanted into mice (120 early breast cancers and 24 metastatic breast cancers). 131 early breast cancer samples and 67 metastatic breast cancer samples were digested for in vitro experiments. Of these, 117 early breast cancer samples and 63 metastatic samples yielded viable cells for experiments. The median number of cells isolated from early breast cancer samples was 35,000 and the median number of cells isolated from metastatic breast cancer samples was 5,250,000.

Cancer stem cell activity was assessed using mammosphere colony formation and self-renewal assays in 117 early breast cancers and 63 metastatic breast cancer cases (Representative images of mammospheres in culture Fig. 1a). Patient-derived samples collected represented all molecular subtypes of breast cancer defined by their oestrogen, progesterone and Her2 receptor expression (Clinical information of all samples used in this study is summarised in Table 1). 
Metastatic tumour cells formed mammospheres (i.e. mammosphere forming efficiency $>0$ ) more frequently than early breast cancers (61/63 (97\%) MBC vs. 90/117 (77\%) EBC; $p=0.0003$ ) (Fig. 1b), and had higher mammosphere formation efficiency $(0.9 \% \mathrm{MBC}$ vs. $0.6 \% \mathrm{EBC} ; p<0.0001)$ (Fig. 1c). Mammosphere self-renewal rates (measured via secondary mammosphere formation) were similar for early and metastatic breast cancers $(0.41 \% \mathrm{EBC}$ vs. $0.29 \% \mathrm{MBC}$, $p=0.074$ ) (Fig. 1d). Tumour initiation in vivo was more frequent in metastatic than early breast cancer samples, with 15 / $24(63 \%)$ metastatic samples forming tumours in mice compared to $46 / 120$ (38\%) early samples ( $p=0.04)$ (Fig. 1e).

Next, we assessed the relationship between mammosphere colony formation / tumour initiation and clinical parameters in both early and metastatic samples. A summary of all samples which formed mammospheres in vitro or tumours in vivo is given in Table 2. Mammosphere formation was higher in metastatic samples from patients with ER negative disease than ER positive disease $(p=0.02)$, but not in early breast cancer samples (Supplementary Fig. 2a, b). No association was seen between mammosphere colony formation and tumour grade or molecular subtype for early or metastatic breast cancers (Supplementary Fig. 2c-f), or between mammosphere colony formation and Nottingham Prognostic Index (derived from tumour size, number of involved lymph nodes and grade. $<3.4=$ good prognosis, $\geq 3.4=$ poor prognosis) in early breast cancers (Supplementary Fig. 2g). Neither mammosphere selfrenewal nor in vivo tumour initiation ability correlated to the above clinical parameters, although the available numbers of cases where these assays were performed were smaller than those examined for primary mammosphere colony formation (self-renewal: $n=36 \mathrm{EBC}$ and $n=21 \mathrm{MBC}$, in vivo tumour initiation: $n=120 \mathrm{EBC}$ and $n=24 \mathrm{MBC}$ ).

90 breast cancer samples were both implanted in vivo and grown as primary mammospheres in vitro to assess correlations between in vivo and in vitro stem cell activity (70 EBC, a

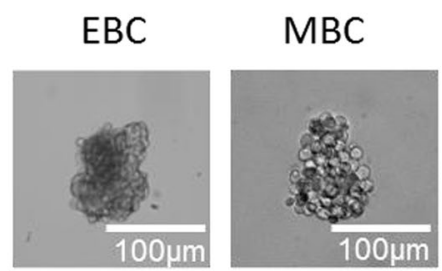

$\mathrm{C}$

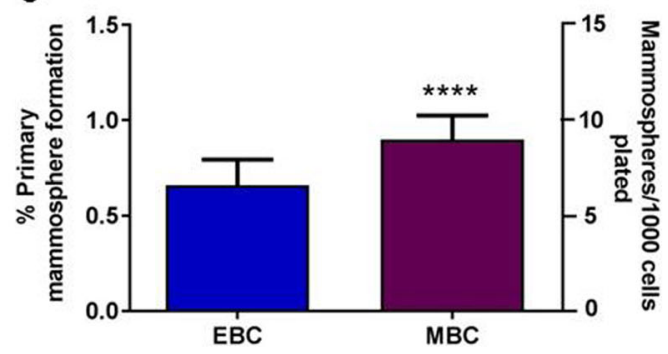

e

Tumour Initiation

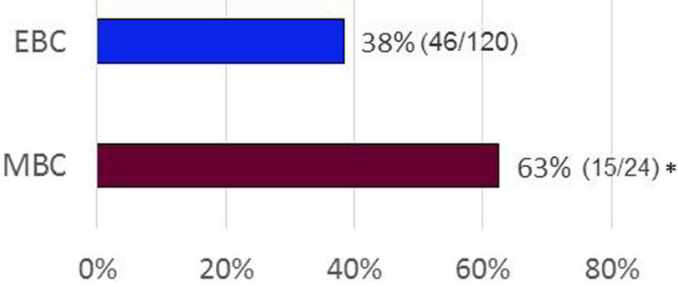

Fig. 1 Breast cancer mammosphere colony formation and in vivo tumour-initiating activity is increased in metastatic compared to early breast cancers. Cells were isolated from breast cancer samples and grown in suspension culture as mammospheres (representative mammospheres from early (EBC) and metastatic (MBC) samples shown in a). Metastatic breast cancer samples were more likely to form mammospheres (MS formation $>0$ ) than early breast cancers cultured under the same conditions $\mathbf{b}$ and had higher primary mammospheres forming efficiencies c (Data presented both as \% mammosphere formation, and b Growth in MS culture

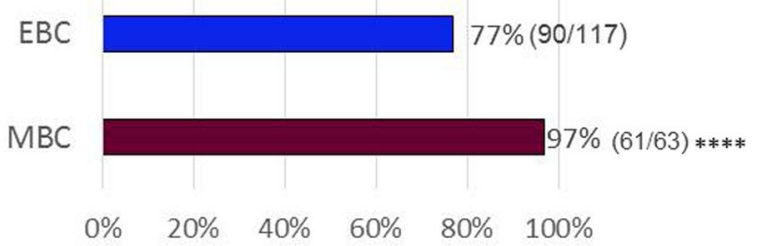

d

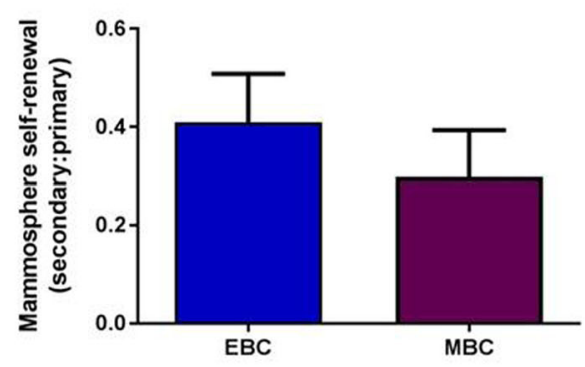

mammospheres formed/1000 cells plated). No difference in secondary mammosphere formation (self-renewal), defined as a ratio of secondary mammospheres: primary mammospheres, was observed between early and metastatic samples d. Metastatic samples had significantly higher in vivo xenotransplantation potential than early breast cancer samples, over an average period of 200 days, irrespective of tumour phenotype $(p=0.04)$, where in vivo growth is defined as tumour formed to size limit $\left(1.3 \mathrm{~cm}^{3}\right) \mathbf{e}$. Data are presented as mean \pm SEM. $* \mathrm{p}<0.05 * * * * \mathrm{p}<0.0005$. Statistical analyses: Chi Squared tests (b and e) and two tailed t-test (c and d) 
$20 \mathrm{MBC})$. Of these, 17 formed tumours when implanted in vivo and 53 formed mammospheres in vitro. Mammosphere colony formation in vitro did not predict tumour initiation in vivo (Supplementary Fig. 2h), and there was no difference in the percentage mammosphere formation in vitro in samples which were able to initiate tumours in vivo (data not shown).

\section{Patient-Derived Xenografts Recapitulate Clinical Tumours}

In total, 144 samples were subcutaneously implanted in vivo into NSG mice (120 EBC, $24 \mathrm{MBC}$ ). Table 2 provides a summary of all samples implanted, and those which formed a tumour in P1, or a stable PDX model. Of the 144 samples implanted, 61 formed tumours in the first passage (P1) in mice (46 EBC, $15 \mathrm{MBC}$ ), and 20 stable PDX tumour models at generation 2 or greater have been established (13 EBC, $7 \mathrm{MBC}$ ). These are derived from $7 \mathrm{ER}+\mathrm{Her} 2-$, 4 ER + Her2+, 1 ER-Her2+ and 8 ER-Her2- patient samples (Table 3 ). Of the 13 early breast cancers from which stable PDX lines were derived, 10 patients were treatment naïve, 2 had received endocrine therapy (BB6RC37 and BB6RC160, both treated with Arimidex) and treatment information was not available for 1 patient (BB2RC08). All 7 of the MBC PDX models were derived from patients who had received prior therapy.

PDX tumours grown in mice resembled patient-derived tumours histologically (Fig. 2a) and retained hormone receptor expression of the patient-derived tumour (Fig. 2b). There was no association between the ability of an early breast cancer sample to form a stable PDX and clinical

Table 2 Summary of samples exhibiting cancer stem cell properties. Clinical data for all samples which formed mammospheres in vitro ( $\mathrm{n}=90 \mathrm{EBC}, \mathrm{n}=61 \mathrm{MBC})$, a tumour in passage 1 in vivo $(n=46 \mathrm{EBC}$, $n=15 \mathrm{MBC})$ or a stable PDX model $(n=13 \mathrm{EBC}, n=7 \mathrm{MBC})$ is presented. characteristics (grade, NPI group, ER/PR status, Her2 status) (data not shown).

\section{Spontaneous Lung Metastasis of PDX Tumour models Correlates with Mammosphere colony Formation}

When subcutaneous tumour growth (P1) was seen, the lymph nodes, bones, lungs and livers were examined for the presence of metastases post-mortem. This totalled 34 models, comprising 25 derived from early breast cancers and 9 derived from metastatic breast cancers. Metastatic cells were not observed in the lymph nodes, bones or livers of any of the mice, however lung metastases were detected in mice from 14/34 (41\%) models (representative H\&E of lung metastases are shown in Fig. $3 \mathrm{a}$, details of mice with lung metastasis are given in Supplementary Table 1). These included 11 of our 20 stable PDX models (detailed in Table 3). Tumours which metastasised to the lung comprised 7 ER + Her2- models, 3 ER + Her2+ models, 1 ER-Her2+ models and 3 ER-Her2-, from 8 early breast cancer and 6 metastatic samples. Human origin of the cells was confirmed using a human-specific mitochondrial antibody (Fig. 3b). Lung metastases retained the hormone receptor status of the primary tumour (Fig. 3c-e shows ER (Fig. 3c), PR (Fig. 3d) and Her2 (Fig. 3e) from an $\mathrm{ER}+, \mathrm{PR}+, \mathrm{Her} 2$ - tumour) and contained Ki67 positive dividing cells (Fig. 3f). Mammosphere colony formation (primary mammosphere generation) by patient-derived tumour cells before implantation into mice predicted the ability of a PDX tumour to metastasise in vivo, with samples with a higher percentage mammosphere forming efficiency being significantly more likely to metastasis to the lungs in PDX models $(p=0.05)$ (Fig. 3g).

Data is presented as successful growth / number of samples tested for each clinical characteristic. ER; oestrogen receptor, PR; progesterone receptor, Her2; Her2 receptor, NPI; Nottingham Prognostic Index. NA; data not available

\begin{tabular}{|c|c|c|c|c|c|c|c|}
\hline & & \multicolumn{2}{|c|}{ Formed Mammospheres } & \multicolumn{2}{|c|}{ Formed tumour in vivo $(\mathrm{P} 1)$} & \multicolumn{2}{|c|}{ Formed stable PDX model } \\
\hline & & $\operatorname{EBC}(n=90)$ & $\operatorname{MBC}(n=61)$ & $\mathrm{EBC}(n=46)$ & $\operatorname{MBC}(n=15)$ & $\operatorname{EBC}(n=13)$ & $\operatorname{MBC}(n=7)$ \\
\hline \multirow[t]{4}{*}{ Grade } & 1 & $3 / 4(75 \%)$ & $3 / 3(100 \%)$ & $1 / 6(17 \%)$ & NA & NA & NA \\
\hline & 2 & $34 / 45(76 \%)$ & $27 / 28(96 \%)$ & $22 / 50(44 \%)$ & $8 / 14(57 \%)$ & $4 / 22(18 \%)$ & $3 / 8(38 \%)$ \\
\hline & 3 & $48 / 63(76 \%)$ & $18 / 18(100 \%)$ & $30 / 59(34 \%)$ & $4 / 6(67 \%)$ & $8 / 20(40 \%)$ & $2 / 4(50 \%)$ \\
\hline & Unknown & $5 / 5(100 \%)$ & $13 / 14(93 \%)$ & $3 / 5(60 \%)$ & $3 / 4(75 \%)$ & $1 / 4(25 \%)$ & $2 / 3(67 \%)$ \\
\hline \multirow[t]{5}{*}{ Hormone receptor status } & ER+Her2- & $50 / 65(77 \%)$ & $46 / 48(96 \%)$ & $26 / 71(36 \%)$ & $10 / 19(53 \%)$ & $2 / 26(8 \%)$ & $5 / 8(63 \%)$ \\
\hline & ER+Her2+ & $12 / 13(92 \%)$ & $3 / 3(100 \%)$ & $4 / 11(36 \%)$ & $1 / 1(100 \%)$ & $3 / 4(75 \%)$ & $1 / 1(100 \%)$ \\
\hline & ER-Her2+ & $6 / 10(60 \%)$ & $1 / 1(100 \%)$ & $4 / 9(44 \%)$ & $1 / 1(100 \%)$ & $1 / 4(25 \%)$ & $0 / 1(0 \%)$ \\
\hline & ER-Her2- & $20 / 25(80 \%)$ & $8 / 8(100 \%)$ & $11 / 26(42 \%)$ & $3 / 3(100 \%)$ & $7 / 11(64 \%)$ & $1 / 3(33 \%)$ \\
\hline & Unknown & $2 / 4(50 \%)$ & $3 / 3(100 \%)$ & $1 / 4(25 \%)$ & $0 / 0(0 \%)$ & $0 / 1(0 \%)$ & $0 / 2(0 \%)$ \\
\hline \multirow[t]{3}{*}{ NPI } & Good $(<3.4)$ & $10 / 12(83 \%)$ & NA & $3 / 13(23 \%)$ & NA & $0 / 2(0 \%)$ & NA \\
\hline & Moderate/Poor $(>3.4)$ & $72 / 95(76 \%)$ & NA & $33 / 91(36 \%)$ & NA & $8 / 22(36 \%)$ & NA \\
\hline & NA & $8 / 10(80 \%)$ & NA & $10 / 16(63 \%)$ & NA & $5 / 22(23)$ & NA \\
\hline
\end{tabular}


Table 3 Summary of Patient-Derived Xenografts created in this study. 20 stable PDX models were created during this study. Receptor status information is presented for clinical tumours and PDX tumours. The highest passage number for each model is presented, as is if PDX models spontaneously metastasise to the lung. EBC; early breast cancer. MBC; metastatic breast cancer. IDC; Invasive Ductal Carcinoma, DCIS; Ductal Carcinoma in situ, NA; data not available, NE; not examined

\begin{tabular}{|c|c|c|c|c|c|c|c|c|c|c|}
\hline \multirow[b]{2}{*}{ Model } & \multirow[b]{2}{*}{ Type } & \multicolumn{4}{|c|}{ Clinical information } & \multicolumn{5}{|c|}{ PDX information } \\
\hline & & Pathology & ER & PR & Her2 & ER & PR & Her2 & Passage & Lung Mets? \\
\hline BB2RC08 & $\mathrm{EBC}$ & NA & Pos & NA & Neg & Pos & Neg & Neg & P4 & Yes \\
\hline BB6RC80 & $\mathrm{EBC}$ & IDC & Pos & Pos & Neg & Pos & Pos & Neg & P3 & NE \\
\hline BB6RC39 & $\mathrm{EBC}$ & IDC & Pos & Pos & Pos & Pos & Pos & Pos & P3 & Yes \\
\hline BB6RC87 & $\mathrm{EBC}$ & IDC/DCIS & Pos & Neg & Pos & NA & NA & NA & P1 & NE \\
\hline BB6RC160 & $\mathrm{EBC}$ & IDC/DCIS & Pos & Neg & Pos & NA & NA & NA & $\mathrm{P} 2$ & Yes \\
\hline BB6RC148 & $\mathrm{EBC}$ & IDC & Neg & Neg & Pos & NA & NA & NA & P3 & Yes \\
\hline BB6RC37 & $\mathrm{EBC}$ & IDC & Neg & $\mathrm{Neg}$ & $\mathrm{Neg}$ & $\mathrm{Neg}$ & Neg & $\mathrm{Neg}$ & P3 & No \\
\hline BB6RC52 & $\mathrm{EBC}$ & IDC & $\mathrm{Neg}$ & Neg & $\mathrm{Neg}$ & NA & NA & NA & P4 & Yes \\
\hline BB6RC69 & $\mathrm{EBC}$ & IDC/DCIS & $\mathrm{Neg}$ & Neg & $\mathrm{Neg}$ & $\mathrm{Neg}$ & Neg & $\mathrm{Neg}$ & P3 & No \\
\hline BB6RC88 & $\mathrm{EBC}$ & IDC & Neg & Pos & $\mathrm{Neg}$ & NA & NA & NA & P2 & Yes \\
\hline BB6RC153 & $\mathrm{EBC}$ & IDC & Neg & $\mathrm{Neg}$ & $\mathrm{Neg}$ & Neg & Neg & Neg & P2 & Yes \\
\hline BB6RC191 & $\mathrm{EBC}$ & NA & Neg & Neg & $\mathrm{Neg}$ & Neg & Neg & Neg & P2 & $\mathrm{NE}$ \\
\hline BB6RC193 & $\mathrm{EBC}$ & IDC & Neg & Neg & Neg & NA & NA & NA & P1 & No \\
\hline BB3RC29 & $\mathrm{MBC}$ & NA & Pos & $\mathrm{Neg}$ & $\mathrm{Neg}$ & $\mathrm{Neg}$ & Neg & $\mathrm{Neg}$ & P9 & Yes \\
\hline BB3RC31 & $\mathrm{MBC}$ & IDC & Pos & Pos & $\mathrm{Neg}$ & Pos & Pos & Neg & P8 & Yes \\
\hline BB3RC32 & $\mathrm{MBC}$ & IDC & Pos & Pos & $\mathrm{Neg}$ & Pos & Pos & $\mathrm{Neg}$ & $\mathrm{P} 4$ & Yes \\
\hline BB3RC50 & $\mathrm{MBC}$ & IDC & Pos & $\mathrm{Neg}$ & $\mathrm{Neg}$ & Pos & Neg & $\mathrm{Neg}$ & P3 & Yes \\
\hline BB3RC72 & $\mathrm{MBC}$ & NA & Pos & Pos & $\mathrm{Neg}$ & NA & NA & NA & $\mathrm{P} 1$ & $\mathrm{NE}$ \\
\hline BB3RC71 & $\mathrm{MBC}$ & IDC & Pos & Pos & Pos & NA & NA & NA & $\mathrm{P} 2$ & $\mathrm{NE}$ \\
\hline BB3RC84 & $\mathrm{MBC}$ & NA & $\mathrm{Neg}$ & $\mathrm{Neg}$ & $\mathrm{Neg}$ & Neg & Neg & Neg & P2 & No \\
\hline
\end{tabular}

Finally we investigated whether any clinical parameters correlated to the ability to metastasise in vivo. PDX tumours established from high grade, early breast cancer samples were more likely to metastasise to the lungs, with 6/9 high grade (Grade 3) tumours metastasising compared to $1 / 10$ low grade tumours (Grade 1 or 2) $(p=0.02)$ (Fig. 3h).

\section{Discussion}

There is evidence to suggest that breast cancer metastases are initiated by stem-like cells [11]. In the current study, we investigated breast CSC activity and metastasis using prospectively collected early and metastatic patient-derived samples in vitro and in vivo. We established that cells derived from metastatic fluids possessed an increased capacity to form both mammospheres in vitro and tumours in vivo compared to early breast cancer samples. Furthermore, we demonstrated that samples which metastasised to the lung in mice were those which possessed significantly higher mammosphere-forming efficiency in vitro.

To our knowledge, this is the largest prospective study of patient-derived tumour mammosphere formation. We compared mammosphere formation to clinical parameters across the whole patient cohort and found no correlation of mammosphere formation to clinical parameters such as tumour grade, molecular subtype or Nottingham Prognostic Index (NPI, derived from tumour size, number of involved lymph nodes and grade). However, higher mammosphere forming efficiency was observed in patients with ERnegative metastatic disease. This supports previous work where expression of the cancer stem cell marker ALDH1 has been shown to be more common in ER- tumours $[13,14]$. In vivo tumour initiation capability did not correspond to the tumour characteristics of tumour grade, molecular subtype, hormone receptor status or NPI. The relationship between tumour characteristics and in vivo engraftment is controversial, with some studies showing hormone receptor status of the primary tumour to predict engraftment [15-18], and others showing no relationship $[19,20]$. Our data adds to those studies suggesting that hormone receptor status does not predict engraftment in vivo.

Throughout this study we have utilised mammosphere culture to assess CSC activity in vitro. It should be noted that there are limitations to this technique. Aggregation of cells can occur, leading to a misinterpretation of results. To minimise cellular aggregation, we have fully optimised our protocols with regard to seeding density, culture time and culture 
conditions [12]. Further, we have previously established that mammospheres can be generated from a single cell [21], and that when single mammospheres are disaggregated and replated at one cell per well, only one mammosphere will form $[7,8]$. These results demonstrate that the mammospheres reported in our study are not a result of cellular aggregation. Despite limitations, the mammosphere assay provides the advantage that it can be performed on a small number of isolated cells. Given the low yields generated from some of our early breast cancer samples (median cell number isolated; 35,000), this assay allowed us to gain an in vitro measure of CSC activity in a far larger number of samples than would have been achievable using FACS-based assays.

We did not observe a correlation between mammosphere formation in vitro and tumour initiation in vivo, our two measures of CSC activity. These have been previously shown to correlate in samples taken from metastatic breast cancer patients [22]. However, although tumour-propagating ability can reflect sphere-forming capacity, they do not always correlate. This is likely to be related to differences in environmental factors in vitro and in vivo. In fact, we previously reported a strong positive correlation between mammosphere formation and tumour initiation in limiting dilution assays once PDX models are established [23].

Patient samples derived from metastases had increased CSC activity compared to early tumours both as mammospheres in vitro and by initiating tumours in vivo. These results contrast to one previous study, which reported no difference between tumour initiation ability between early and metastatic samples [19], but support recent work demonstrating that recurrent tumours have a higher engraftment rate in vivo [18]. The increased CSC activity observed in metastatic samples suggests that CSCs are those which are able to survive conventional treatment and become metastatic in breast cancer patients. This supports results of two previous studies. Firstly it has been demonstrated that high expression of CD44+/CD24- CSCs in tumours favours distant metastases [24], and secondly patient treatment with chemotherapy has been shown to increase CSC activity (as assessed by CD44+/CD24- expression and mammosphere forming efficiency) in patientderived tumours [25]. As all of the patients in our metastatic cohort had been exposed to chemotherapy, this may provide an explanation for the increase in CSC activity in this group. It is unknown if chemotherapy promotes the expansion of CSCs, or if CSCs are selected by chemotherapy based on their intrinsic drug resistance. A further hypothesis is that metastatic niche selectively supports the growth of cancer stem cells resulting in an expansion of their numbers in the metastatic setting. Although the mechanism remains unclear, our findings emphasise the importance of developing anti-CSC treatments to prevent and treat metastatic disease. a

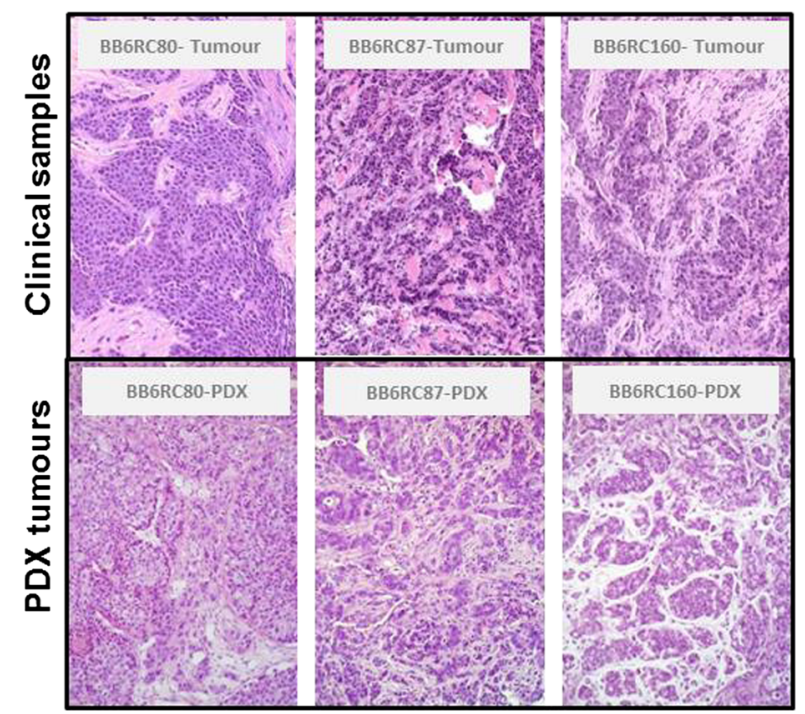

b

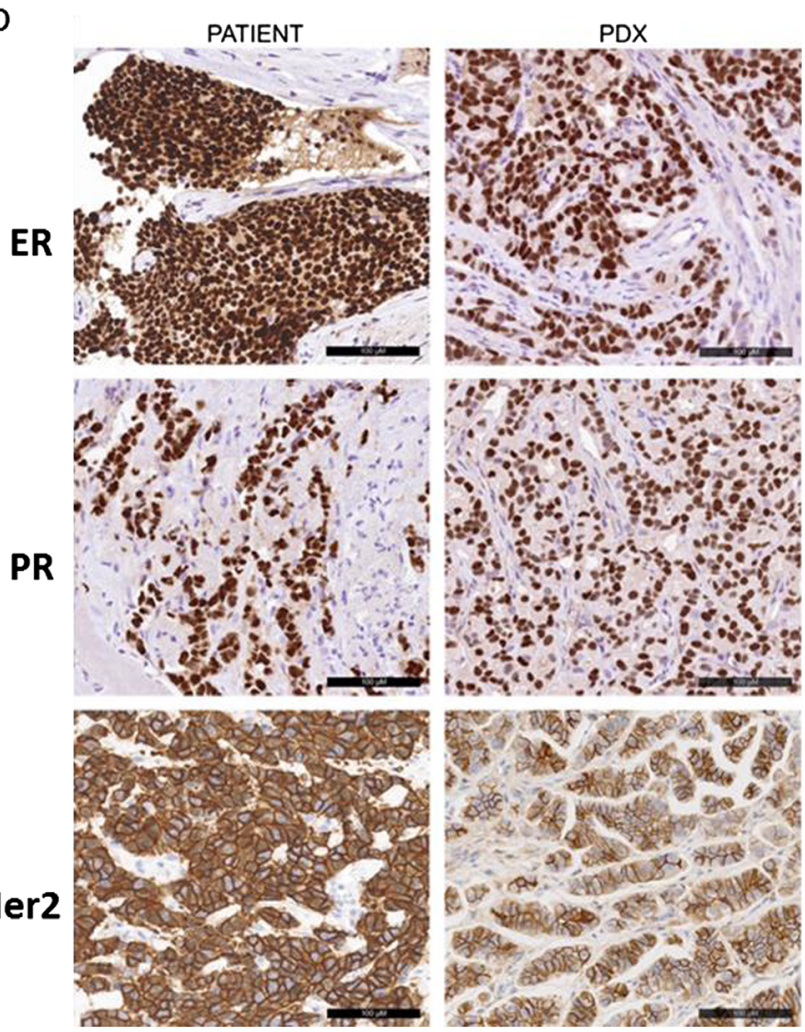

Fig. 2 Characterisation of Patient-Derived Xenograft tumours. Of the 144 tumours implanted in this study, 61 grew in the first passage in mice and 20 stable PDX models were created. Comparisons of representative patient tumour samples and their corresponding patientderived xenograft in passage 1 a. Patient-derived xenografts retained ER, PR and Her2 status of the patient tumour. Representative images in b. Scale bar $=100 \mathrm{uM}$

In our study, we prospectively derived 20 stable breast cancer patient-derived xenograft (PDX) models from 144 implanted tumours. Importantly, 11 of 20 were derived from ER positive tumours, a subtype which has historically been more 
Fig. 3 Mammosphere colony formation predicts metastasis in PDX models. Lung metastases were detected in 14/34 models where a tumour formed in passage 1 by $\mathrm{H} \& \mathrm{E}$ a and confirmed by staining with a human specific mitochondrial antibody b. Lung metastases retained the ER $\mathbf{c}, \mathrm{PR} \mathbf{d}$ and $\mathrm{Her} 2$ e status of the primary tumour and contained ki67 positive dividing cells f. Primary mammosphere formation predicted PDX metastases, with samples which metastasised to the lungs in vivo having a significantly higher \% mammosphere forming efficiency in vitro $\mathbf{g}$. Metastases are more likely to form in PDX models from high grade EBC tumours (grade 3 ) than low grade (grade 1 and 2) h. Data are presented as mean \pm SEM. $* p<0.05$ Statistical analysis: Mann -Whitney U Test
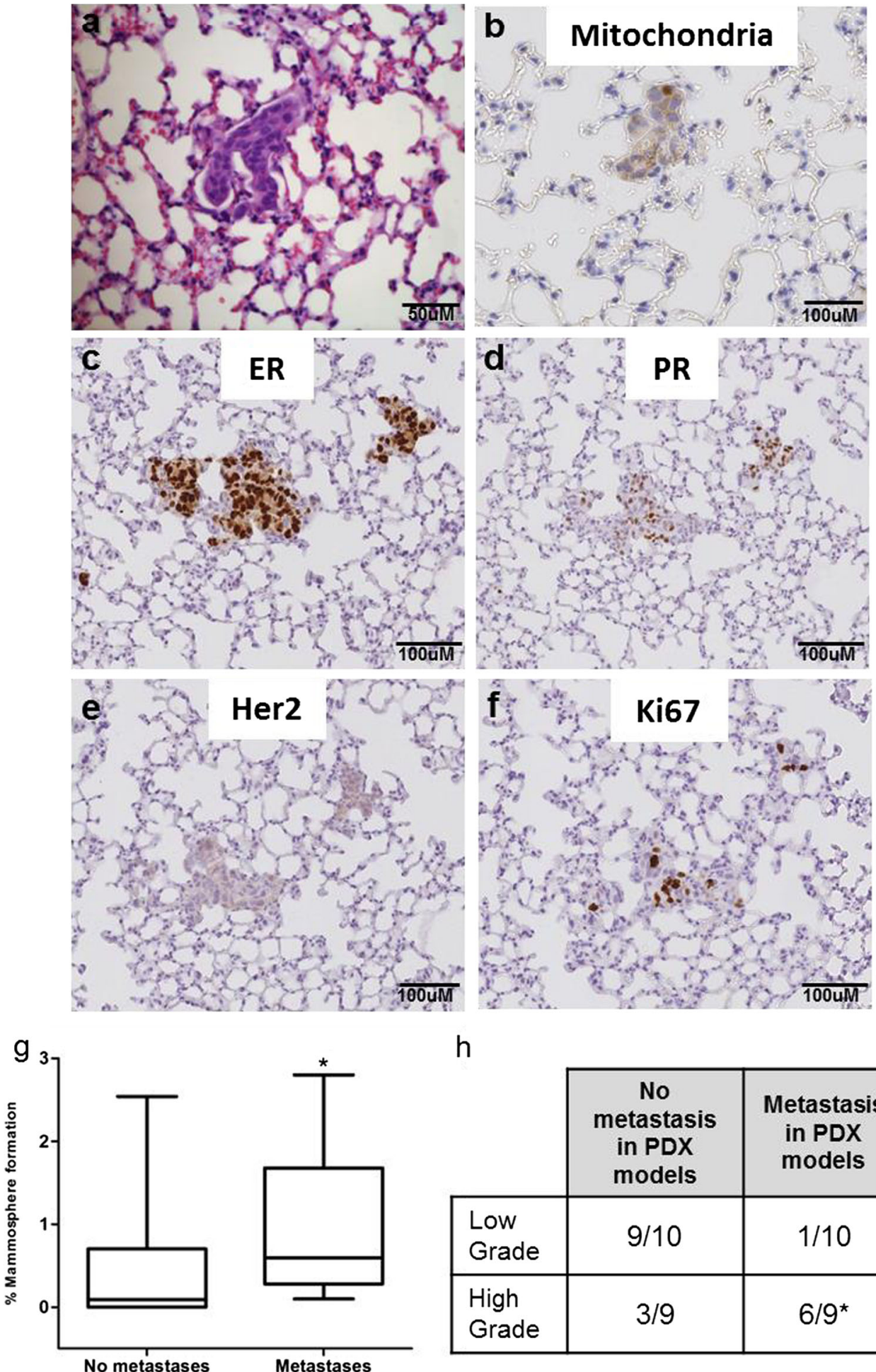

$\mathrm{h}$

\begin{tabular}{|l|c|c|}
\cline { 2 - 3 } \multicolumn{1}{c|}{} & $\begin{array}{c}\text { No } \\
\text { metastasis } \\
\text { in PDX } \\
\text { models }\end{array}$ & $\begin{array}{c}\text { Metastasis } \\
\text { in PDX } \\
\text { models }\end{array}$ \\
\hline $\begin{array}{l}\text { Low } \\
\text { Grade }\end{array}$ & $9 / 10$ & $1 / 10$ \\
\hline $\begin{array}{l}\text { High } \\
\text { Grade }\end{array}$ & $3 / 9$ & $6 / 9^{\star}$ \\
\hline
\end{tabular}

difficult to successfully engraft [26]. Where we assessed spontaneous metastases in the models that formed tumours in the first generation, 14/34 (41 \%) developed lung metastases, similar to previously published studies where 38/ $70(54 \%)$ PDX models were reported to develop lung metastases $[15,16,19,20,27]$.
We are the first to assess metastatic capability in PDX models in relation to CSC frequency, and show that tumours which metastasise to the lung are those which have significantly higher CSC activity in vitro. Supporting this finding, a recent study demonstrated that single cells which metastasise in PDX models possess a gene signature similar to stem cells 
[11]. A limitation of studying metastasis in PDX models is that the frequency and sites of metastases in PDX models may differ from the patient [28]. Follow up data at 5 or more years post diagnosis will be important to help determine if metastases in our models correspond to that in the patients from which they were generated. Independent of this, these models will provide a useful biological resource for the breast cancer community, and will now be used as a platform to test new breast cancer anti-metastasis therapies.

In summary, both in vitro and in vivo CSC activities are increased in metastatic samples, and CSC activity in vitro predicts metastasis in vivo. These results suggest that breast CSC activity may predict for poor outcome tumours and that antiCSC treatment should be utilised in the prevention and treatment of breast metastasis. Further validation of these results is now required, both in larger patient/PDX cohorts and by assessing patient follow-up data.

Acknowledgments We would like to thank all patients who donated tissue to this study, and the Manchester Cancer Research Centre Biobank for consenting patients and collecting tissue.

\section{Compliance with Ethical Standards}

Funding This study was funded by Cancer Research UK and Breast Cancer Now. MK was funded by S cience and Technology Development Fund fellowship No. 6123.

Research involving Human Participants All patients underwent fully informed consent in accordance with local research ethics committee guidelines. Ethical approval for metastatic samples was granted by the Central Office for Research Ethics Committee study number 05/Q1402/ 25. Early breast cancer samples were collected via the Manchester Cancer Research Centre Biobank which is licensed by the Human Tissue Authority (licence number: 30,004) and has been ethically approved as a research tissue bank by the South Manchester Research Ethics Committee (Ref: 07/H1003/161 + 5).

Conflict of Interest The authors declare that they have no conflict of interest.

Open Access This article is distributed under the terms of the Creative Commons Attribution 4.0 International License (http:// creativecommons.org/licenses/by/4.0/), which permits unrestricted use, distribution, and reproduction in any medium, provided you give appropriate credit to the original author(s) and the source, provide a link to the Creative Commons license, and indicate if changes were made.

\section{References}

1. O'Shaughnessy J. Extending survival with chemotherapy in metastatic breast cancer. Oncologist. 2005;10(Suppl 3):20-9. doi:10.1634/theoncologist.10-90003-20.

2. Chambers AF, Groom AC, MacDonald IC. Dissemination and growth of cancer cells in metastatic sites. Nat Rev Cancer. 2002;2(8):563-72. doi:10.1038/nrc865.

3. Al-Hajj M, Wicha MS, Benito-Hernandez A, Morrison SJ, Clarke MF. Prospective identification of tumorigenic breast cancer cells.
Proc Natl Acad Sci U S A. 2003;100(7):3983-8. doi:10.1073 /pnas.0530291100.

4. Bonnet D, Dick JE. Human acute myeloid leukemia is organized as a hierarchy that originates from a primitive hematopoietic cell. Nat Med. 1997;3(7):730-7.

5. Reynolds BA, Weiss S. Generation of neurons and astrocytes from isolated cells of the adult mammalian central nervous system. Science. 1992;255(5052):1707-10.

6. Dontu G, Abdallah WM, Foley JM, Jackson KW, Clarke MF, Kawamura MJ, Wicha MS. In vitro propagation and transcriptional profiling of human mammary stem/progenitor cells. Genes Dev. 2003;17(10):1253-70.

7. Farnie G, Clarke RB, Spence K, Pinnock N, Brennan K, Anderson $\mathrm{NG}$, et al. Novel cell culture technique for primary ductal carcinoma in situ: role of Notch and epidermal growth factor receptor signaling pathways. J Natl Cancer Inst. 2007;99(8):616-27. doi:10.1093 jinci/djk133.

8. Harrison H, Farnie G, Howell SJ, Rock RE, Stylianou S, Brennan $\mathrm{KR}$, et al. Regulation of breast cancer stem cell activity by signaling through the Notch4 receptor. Cancer Res. 2010;70(2):709-18. doi:10.1158/0008-5472.CAN-09-1681.

9. Charafe-Jauffret E, Ginestier C, Iovino F, Wicinski J, Cervera N, Finetti $\mathrm{P}$, et al. Breast cancer cell lines contain functional cancer stem cells with metastatic capacity and a distinct molecular signature. Cancer Res. 2009;69(4):1302-13. doi:10.1158/0008-5472. CAN-08-2741.

10. Croker AK, Goodale D, Chu J, Postenka C, Hedley BD, Hess DA, et al. High aldehyde dehydrogenase and expression of cancer stem cell markers selects for breast cancer cells with enhanced malignant and metastatic ability. J Cell Mol Med. 2009;13(8B):2236-52. doi:10.1111/j.1582-4934.2008.00455.x.

11. Lawson DA, Bhakta NR, Kessenbrock K, Prummel KD, Yu Y, Takai K, et al. Single-cell analysis reveals a stem-cell program in human metastatic breast cancer cells. Nature. 2015;526(7571):1315. doi:10.1038/nature15260.

12. Shaw FL, Harrison H, Spence K, Ablett MP, Simoes BM, Farnie G, et al. A detailed mammosphere assay protocol for the quantification of breast stem cell activity. J Mammary Gland Biol Neoplasia. 2012;17(2):111-7. doi:10.1007/s10911-012-9255-3.

13. Park SY, Lee HE, Li H, Shipitsin M, Gelman R, Polyak K. Heterogeneity for stem cell-related markers according to tumor subtype and histologic stage in breast cancer. Clin Cancer Res: Off J American Assoc Cancer Res. 2010;16(3):876-87. doi:10.1158/1078-0432.CCR-09-1532.

14. Ginestier C, Hur MH, Charafe-Jauffret E, Monville F, Dutcher J, Brown $\mathrm{M}$, et al. ALDH1 is a marker of normal and malignant human mammary stem cells and a predictor of poor clinical outcome. Cell Stem Cell. 2007;1(5):555-67. doi:10.1016/j. stem.2007.08.014.

15. Marangoni E, Vincent-Salomon A, Auger N, Degeorges A, Assayag F, de Cremoux P, et al. A new model of patient tumorderived breast cancer xenografts for preclinical assays. Clin Cancer Res: Off J American Assoc Cancer Res. 2007;13(13):3989-98. doi:10.1158/1078-0432.CCR-07-0078.

16. Zhang X, Claerhout S, Prat A, Dobrolecki LE, Petrovic I, Lai Q, et al. A renewable tissue resource of phenotypically stable, biologically and ethnically diverse, patient-derived human breast cancer xenograft models. Cancer Res. 2013;73(15):4885-97. doi:10.1158 /0008-5472.CAN-12-4081.

17. Petrillo LA, Wolf DM, Kapoun AM, Wang NJ, Barczak A, Xiao Y, et al. Xenografts faithfully recapitulate breast cancerspecific gene expression patterns of parent primary breast tumors. Breast Cancer Res Treat. 2012;135(3):913-22. doi:10.1007/s10549-012-2226-y.

18. Moon HG, Oh K, Lee J, Lee M, Kim JY, Yoo TK, et al. Prognostic and functional importance of the engraftment-associated genes in 
the patient-derived xenograft models of triple-negative breast cancers. Breast Cancer Res Treat. 2015;154(1):13-22. doi:10.1007 /s10549-015-3585-y.

19. DeRose YS, Wang G, Lin YC, Bernard PS, Buys SS, Ebbert MT, et al. Tumor grafts derived from women with breast cancer authentically reflect tumor pathology, growth, metastasis and disease outcomes. Nat Med. 2011;17(11):1514-20. doi:10.1038/nm.2454.

20. Visonneau S, Cesano A, Torosian MH, Miller EJ, Santoli D. Growth characteristics and metastatic properties of human breast cancer xenografts in immunodeficient mice. Am J Pathol. 1998;152(5):1299-311.

21. Singh JK, Farnie G, Bundred NJ, Simoes BM, Shergill A, Landberg $\mathrm{G}$, et al. Targeting CXCR1/2 significantly reduces breast cancer stem cell activity and increases the efficacy of inhibiting HER2 via HER2-dependent and -independent mechanisms. Clin Cancer Res: Off J American Assoc Cancer Res. 2013;19(3):643-56. doi:10.1158/1078-0432.CCR-12-1063.

22. Grimshaw MJ, Cooper L, Papazisis K, Coleman JA, Bohnenkamp HR, Chiapero-Stanke L, et al. Mammosphere culture of metastatic breast cancer cells enriches for tumorigenic breast cancer cells. Breast Cancer Res: BCR. 2008;10(3):R52. doi:10.1186/bcr2106.

23. Simoes BM, O'Brien CS, Eyre R, Silva A, Yu L, SarmientoCastro A, et al. Anti-estrogen Resistance in Human Breast
Tumors Is Driven by JAG1-NOTCH4-Dependent Cancer Stem Cell Activity. Cell Rep. 2015;12(12):1968-77. doi:10.1016/j.celrep.2015.08.050.

24. Abraham BK, Fritz P, McClellan M, Hauptvogel P, Athelogou M, Brauch $\mathrm{H}$. Prevalence of CD44+/CD24-/low cells in breast cancer may not be associated with clinical outcome but may favor distant metastasis. Clin Cancer Res: Off J American Assoc Cancer Res. 2005;11(3):1154-9.

25. Li X, Lewis MT, Huang J, Gutierrez C, Osborne CK, Wu MF, et al. Intrinsic resistance of tumorigenic breast cancer cells to chemotherapy. J Natl Cancer Inst. 2008;100(9):672-9. doi:10.1093 /jnci/djn123.

26. Cottu P, Marangoni E, Assayag F, de Cremoux P, Vincent-Salomon $\mathrm{A}$, Guyader C, et al. Modeling of response to endocrine therapy in a panel of human luminal breast cancer xenografts. Breast Cancer Res Treat. 2012;133(2):595-606. doi:10.1007/s10549-011-1815-5.

27. Liu H, Patel MR, Prescher JA, Patsialou A, Qian D, Lin J, et al. Cancer stem cells from human breast tumors are involved in spontaneous metastases in orthotopic mouse models. Proc Natl Acad Sci U S A. 2010;107(42):18115-20. doi:10.1073/pnas. 1006732107.

28. Whittle JR, Lewis MT, Lindeman GJ, Visvader JE. Patient-derived xenograft models of breast cancer and their predictive power. Breast Cancer Res: BCR. 2015;17:17. doi:10.1186/s13058-015-0523-1. 